\title{
Supervoxel based point cloud segmentation algorithm (Withdrawal Notice)
}

Fujing Tian, Hua Chen, Mei Yu, Ken Chen, Gangyi Jiang

Fujing Tian, Hua Chen, Mei Yu, Ken Chen, Gangyi Jiang, "Supervoxel based point cloud segmentation algorithm (Withdrawal Notice)," Proc. SPIE 11187, Optoelectronic Imaging and Multimedia Technology VI, 111870Y (18 November 2019); doi: 10.1117/12.2538691

SPIE. Event: SPIE/COS Photonics Asia, 2019, Hangzhou, China 


\title{
Supervoxel based point cloud segmentation algorithm (Withdrawal Notice)
}

\author{
Fujing Tian, ${ }^{1}$ Hua Chen, ${ }^{1}$ Mei $Y u,{ }^{1,2}$ Ken Chen, ${ }^{1}$ Gangyi Jiang ${ }^{1,2}$ \\ ${ }^{1}$ Ningbo Univ. (China) \\ ${ }^{2}$ Nanjing Univ. (China) \\ Proceedings Volume 11187, Optoelectronic Imaging and Multimedia Technology VI; 111870Y (2019) \\ https://doi.org/10.1117/12.2538691 \\ Event: SPIE/COS Photonics Asia, 2019, Hangzhou, China
}

Online Publication Date: 18 November 2019

Withdrawn from Publication: 26 February 2020

Publisher's Note: This paper, originally published on 18 November 2019, was withdrawn 26 February 2020 for ethics violations. 